\section{TAKING TEETH TOOLS INTO SCHOOLS}

The County Health Partnerships Oral Health Promotion Team (part of Nottinghamshire

Healthcare) has developed an initiative called 'Teeth Tools for Schools', a dynamic

teaching resource for primary schools in an area where just under a half of

all five-year-olds have at least one decayed, missing or filled baby tooth

(compared to just over a third in England).

Teeth Tools for Schools is an oral health resource for teaching staff

working with children aged three to 11 , created to

support planned educational activities and raise the importance of oral health. The pack contains detailed information on the basics of oral health; easy to follow lesson plans; interactive activities for children to explore and gain knowledge; ideas to involve individuals, classes and whole school approaches; and promotional materials for use with children and parents.

All primary schools in Nottingham City, Nottinghamshire and Bassetlaw have received their own copy of the Teeth Tools for Schools pack.

Oral Health Promotion coordinator Julia Wilkinson, who appeared on the cover of Vital in 2005 wielding a giant toothbrush (see page 28 of this issue) hopes to inspire other regions of the UK to adopt similar initiatives. 'The feedback has been excellent,' said Julia. 'We can now proudly say that all our schools and teachers are sharing the consistent oral health messages and they are doing so with fun, interactive sessions that link to the National Curriculum.'

Teeth Tools for Schools joins Nottinghamshire Healthcare's well established City Smiles and Incredible Mouths programmes, which promote key oral health messages through partnership working with health visitors, midwives and school health teams.

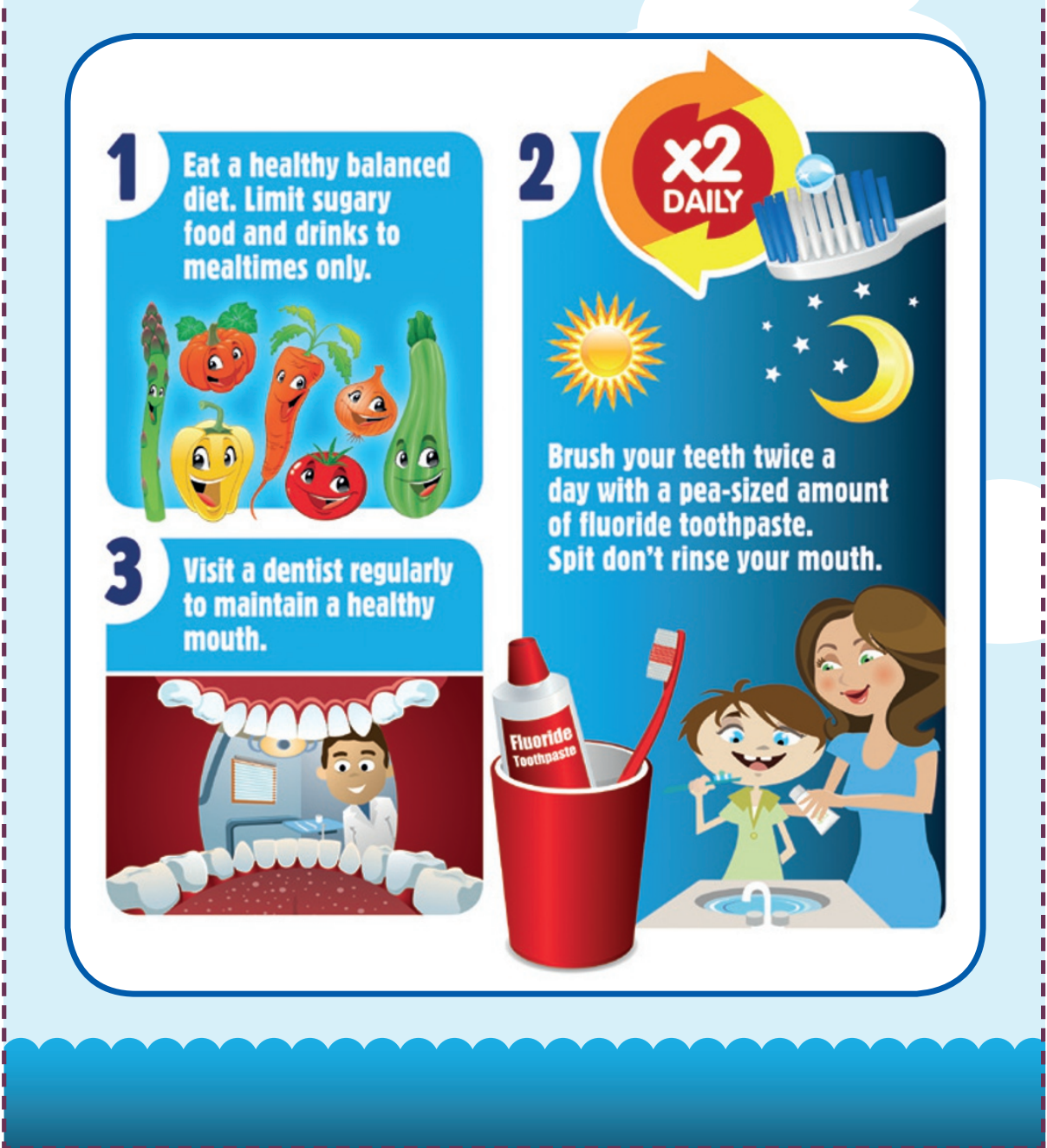

LONDON PRACTICE TAKES CPD TRAINING INTO THEIR \section{OWN HANDS}

A dental practice in Thamesmead, London has come up with their own strategy for relaying important practice policies and protocol to the whole team and at the same time meeting the General Dental Council's (GDC's) continuing orofessional
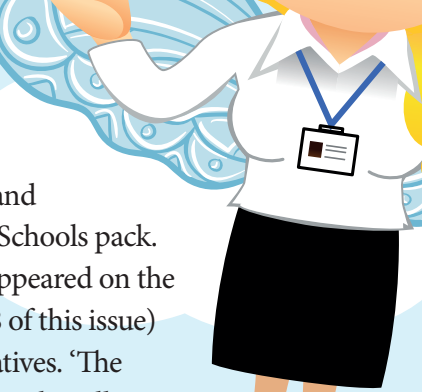
3

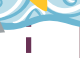

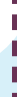

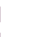
I I satisfied that we could achieve the criteria, the solution was to take a policy/protocol or regulation, read through and draw out questions which would meet the GDC requirements.' An answer sheet was created for staff to complete, making marking the whole process fast and efficient.

The staff at Gallions Reach were given an introduction to a topic and provided with a handout to read and answer. Each topic was allocated an hour but staff were given a week to return their completed answer sheets, giving them time to approach Andrew or Jonathan with any questions they might have. Once marked, verifiable CPD certificates were distributed at the next practice meeting.

Andrew continues: 'to measure quality control [we] survey the staff and audit the questionnaire sheets. This has enabled Andrew and Jonathan to refine the way in which they construct their in-house training, meeting the team's and the practice's needs.

Gallions Reach dental nurse Gemma Shove said: 'I think the training is extremely worthwhile. I like the way it is broken down into easy to understand sections. The relaxed way it is presented to us allows me to understand the topic plus we get $\mathrm{CPD}$ and understand the CQC outcomes as well.'

Practices interested in setting up a similar system can email gallions-reach@ btconnect.com for a free guide. 